\title{
Software de ingeniería especializado en el diseño y simulación de plantas de tratamiento de agua residual: revisión
}

\author{
Specialized engineering software for the design and \\ simulation of wastewater treatment plants: A review
}

\author{
J. E. Sánchez Ramírez ${ }^{[1]}$, A. Mejía Fajardo ${ }^{[2]}$ y C. M. Amorocho Cruz ${ }^{[3]}$
}

\begin{abstract}
Resumen
Este documento presenta una revisión sobre los programas más utilizados para la simulación, diseño y control de plantas de tratamiento de agua residual. Se plantea la posibilidad de utilizar este tipo de herramientas para investigación, ambientes profesionales y de docencia. Este documento recopila el software más utilizado, teniendo en cuenta que estas herramientas permiten mejorar el rendimiento, control y la optimización de las plantas de tratamiento de agua residual.
\end{abstract}

Palabras clave: modelación; ASM1; procesos; agua residual; fangos activados; biotecnología.

\begin{abstract}
This paper presents a review of the most frequently used programs for the simulation, design and control of wastewater treatment plants. We set out the possibility of using such tools in research, professional environments and teaching. This document compiles the most frequently used software bearing in mind that such tools improve performance, control and the optimization of the waste water treatment plants.
\end{abstract}

Key words: modeling, ASM1, processes, wastewater, activated sludge, biotechnology.

\section{Introducción}

Utilizar software especializado para realizar la simulación de los procesos de una planta de tratamiento de agua residual constituye una decisión muy importante. Para el uso del software deberá establecerse para qué y quién la usará, deberá decidirse cual utilizar dentro de la gama existente en el mercado y deberá conocerse los alcances del mismo. Estas aplicaciones permiten al usuario manejar varias unidades de proceso relacionadas entre sí, de acuerdo a un diagrama de la planta que el usuario establece. Permitiéndole probar el comportamiento de estas unidades según las condiciones ambientales, de vertido y la legislación aplicada.

[1] MSc. Investigador. Universidad Surcolombiana. Grupo de investigación USCO agroindustria. Javier.E.Sanchez@uv.es.

[2] MSc. Estudiante de doctorado. Dpto. IHIMA U. Politécnica de Valencia (España). Camí de Vera. Pime130@hotmail.com.

[3] PhD profesor. Universidad Surcolombiana. Grupo de investigación USCO agroindustria. claudiamilena.amorocho@ usco.edu.co

Recibido: 3 mayo 2015 - Aceptado: 24 mayo 2015 
Cada aplicación íntegra unos modelos matemáticos que describen el comportamiento de los procesos (Water Treatment Hanbook, 1991). Dentro de los modelos más utilizados se encuentran los desarrollados por la International Water Association (IWA).

El software de simulación permite a los usuarios probar diferentes configuraciones según la planta de tratamiento que se desee simular (Iturrate et al., 2005). Poseen herramientas potentes con el fin de probar diferentes grados de complejidad. Las aplicaciones incorporan gráficas y tablas de información robustas con presentación de resultados fácil de interpretar por el usuario. Sin embargo todas las aplicaciones existentes esconden detrás de su entorno amigable y fácil de usar, la dificultad de usar correctamente todas las herramientas. Se necesita un alto conocimiento de los procesos, los modelos utilizados y de los parámetros necesarios para iniciar la simulación con el fin de obtener los mejores resultados (Olsson, G et al., 1999).

Existen varios tipos de estas aplicaciones que van desde aplicaciones de formación o académicas, hasta herramientas de modelación y simulación. Se encuentran simuladores claramente orientados a ambientes profesionales, con precios más elevados, pero que ofrecen documentación completa, incluso cursos de formación. Dentro de estos ambientes en función de las necesidades y usuarios finales existen dos tipos de simuladores: los de propósito general y los específicos. Los más utilizados son los de propósito general, ya que permiten diseñar el esquema de la planta de tratamiento a modelar. Además permiten en función de las librerías adquiridas o disponibles, utilizar diferentes modelos para cada proceso planteado en el esquema de tratamiento.

Este documento pretende mencionar el software existente más utilizado para el diseño y simulación de plantas de tratamiento de agua residual, desde un punto de vista informativo. Todas las aplicaciones mencionadas presentan funciones específicas y el uso de cada una de ellas depende de la finalidad y los resultados que se quieran obtener.

\section{Revisión de la literatura}

La modelación de procesos tiene como objetivos alcanzar un entendimiento común con el menor esfuerzo posible, poder realizar un análisis del funcionamiento actual del sistema, mejorar la calidad de los procesos y optimizar el funcionamiento. En la actualidad, existe una grande necesidad de difusión del tema y actualización de los modelos existentes. También hay retos bien identificados para la investigación en los aspectos de caracterización y modelación de mecanismos bioquímicos, así como la simplificación de los modelos y los protocolos de simulación (Morenilla et al., 2008; Martínez, et al., 2008).

Según Van Loosdrecht et al. 1999 los modelos son una descripción matemática de una serie de procesos químico, físicos y biológicos que ocurren en un espacio delimitado (tanques y reactores). Sin embargo, el hecho de que un modelo se ajuste bien a los datos experimentales y que prediga bien el comportamiento del sistema estudiado, no significa que los mecanismos del modelo son correctos desde el punto de vista microbiológico.

La International Association on Water Quality (IAWQ) en 1982, conformo un grupo de estudio encargado de desarrollar modelos matemáticos que permitieran expresar los diversos procesos biológicos existentes en las plantas de tratamiento. Además de generar un entorno generalizado mediante modelos simples ("Task Group on Mathematical Modelling for Design and Operation of Activated Sludge Process"). Hasta el momento los modelos más utilizados son los propuestos por la International Water Association (IWA) desde el (ASM1- activated sludge model 1) (Henze et al., 2000), hasta todos los modelos de la serie ASM de 1987,1995, 1999 y el modelo de digestión anaerobia (ADM1-anaerobic digestión model 1). Rivera-Salvador et al. 2009 y Batstone., (2002) describen en detalle el modelo ADM, Hinken et al. 2014 realizan una modificación del modelo ADM1 para emplearlo en la simulación de reactores UASB. El modelo ASM1 fue el primer modelo y la base de numerosas extensiones. Muchos de los conceptos básicos del ASM1 se adaptan a partir del modelo de lodos activados definido por Dold y colegas ( Dold et al., 1980 ).

Hoy en día, el modelo ASM1 sigue siendo ampliamente utilizado ya que permite la simulación del proceso de fangos activados ( Dircks et al., 2001; Roeleveld y van Loosdrecht, 2002; Fang et al., 2011 ). Otras estrategias de simulación que permite simplificar mediante técnicas computacionales el modelo ASM1 han sido descritas por Anderson et al., 2000. Copp, 2002 menciona las experiencias obtenidas en la implementación del modelo ASM1 en diferentes plataformas de software. La descripción detallada del modelo ASM1 así 
como la explicación sobre la matriz utilizada fue descrita por Henze en 1987 (Henze et al., 1987).

En 1995, una versión actualizada del modelo ASM1 fue presentada por Henze como el modelo ASM2. Este modelo incorpora la eliminación biológica de fósforo (Henze et al., 1995). Existen otras extensiones de estos modelos que incluyen una mejor predicción de los procesos de eliminación de nitrógeno, fosforo y procesos físico-químicos (Smolders et al., 1995). Una extensión del modelo ASM2d es el modelo (BNMR1-Biological nutrient removal model 1) descrito por Seco et al., 2004, donde se incluyen los procesos biológicos y fisicoquímicos más importantes en el diseño y simulación de plantas de tratamiento. Otras extensiones han sido reportadas por Roeleveld and van Loosdrecht, 2002 utilizando como punto de partida el modelo ASM1 e incorporándolo a diversas plataformas de simulación. La Tabla 1 muestra un resumen de los principales modelos que han sido mencionados anteriormente y el número aproximado de procesos incluidos en cada uno de ellos.
En el tratamiento de agua, los modelos se expresan a través de balances de masas que toman en cuenta la cinética de reacción de procesos y la hidrodinámica (Metcalf y Eddy. (1991)). La escritura de estos balances resulta en sistemas de ecuaciones diferenciales de difícil resolución, siendo necesario el empleo de algún programa o software. Este tipo de software o de plataformas especiales son llamados simuladores.

Los simuladores son herramientas utilizadas ampliamente (Ayesa et al., 2007; Palma-Acosta et al., 2011; Grau-Gumbau, 2011), poderosas para el diseño, planificación, análisis de procesos, educación y formación. Algunos de los simuladores más utilizados se presentan en la Tabla 2, estos programas incluyen un modelo biológico basado en los modelos desarrollados por la IWA. Incluyen el modelo biológico de lodos activados, algunos incorporan modelos de simulación de sedimentadores, reactores anaerobios y algoritmos de control de oxígeno disuelto (Henry M., 2003). Los simuladores en muchas ocasiones son capaces de

Tabla 1. Procesos incluidos en los modelos más utilizados (Gernaey et al., 2004).

\begin{tabular}{lccc}
\hline Modelos & Nitrificación desnitrificación & Eliminación biológica de P & Procesos \\
\hline ASM1 & $\mathrm{X}$ & & 8 \\
ASM2 & $\mathrm{X}$ & $\mathrm{X}$ & 19 \\
ASM2d & $\mathrm{X}$ & $\mathrm{X}$ & 21 \\
ASM3 & $\mathrm{X}$ & $\mathrm{X}$ & 12 \\
ASM3+BioP & $\mathrm{X}$ & $\mathrm{X}$ & 23 \\
B\&D & $\mathrm{X}$ & $\mathrm{X}$ & 36 \\
TUDP & $\mathrm{X}$ & $\mathrm{X}$ & 21 \\
ASM2d+pH & $\mathrm{X}$ & & 23 \\
ASM1+pH & $\mathrm{X}$ & $\mathrm{X}$ & 10 \\
BNRM1 & $\mathrm{X}$ & & 25 \\
\hline
\end{tabular}

Tabla 2. Simuladores existentes para el diseño y simulación de plantas de tratamiento de agua residual.

\begin{tabular}{lcc}
\hline Simulador & Creador & Origen \\
\hline ASIM & EAWAG & Suiza \\
DESASS & Calagua group- UPV & España \\
BIOWIN & Envirosim Associates L.d & Canadá \\
EFOR & DHI.Inc & Dinamarca \\
GPS-X & Hydromantis Inc. & Canadá \\
SIMBA & IFAK-System Gmbh & Alemania \\
STOAT & WRc Group & Reino unido \\
WEST & Hemmin N.V & Bélgica \\
EDAR 1.0 & CEIT-Cadagua & España \\
AQUASIM & EAWAG & Suiza \\
\hline
\end{tabular}


predecir varios parámetros de calidad partiendo del influente de las estaciones depuradoras. Eriksson et al. (2001) aplico un modelo que le permitió predecir la carga en términos de DQO del influente y relacionarla con la DQO de salida de una planta de tratamiento de la industria del papel.

A continuación se mencionan las características más significativas de cada uno los simuladores mencionados anteriormente. El objetivo de este documento es mostrar los simuladores existentes e ilustrar algunas de las características básicas de las diferentes aplicaciones tales como, aspecto del entorno, una idea rápida sobre su manejo y su aspecto gráfico.

\subsection{ASIM}

Este programa de simulación tiene un nombre que responde al acrónimo de ASIM (Activated Sludge Simulation Program). La versión más difundida y conocida es la 3.0 de agosto de 1994. Fue desarrollado por el Profesor Willi Gujer, estando en el Swiss Federal Institute for Water Resources and Water Pollution Control, EAWAG, Dübendorf. Suiza. En esta aplicación los modelos biocinéticos pueden ser libremente definidos, almacenados y editados por el usuario. Esto permite al investigador desarrollar su propio modelo específico y permite al estudiante aprender gradualmente al poder ir incrementando la complejidad de los modelos. La versión distribuida incluye los modelos ASM1 y ASM2 de la IWA. Así mismo acompaña un modelo para "bulking filamentoso” y otros más. Trabaja bajo el entorno de
MS DOS, lo que supone una desventaja grafica frente a otro tipo de simuladores (Gujer et al., 1995).

\subsection{DESASS}

DESASS es un simulador de plantas de tratamiento potente desarrollado bajo un entorno de Windows mediante el uso de Microsoft visual basic 6.0, capaz de ayudar al diseño, la actualización, la simulación y la optimización de plantas de tratamiento (Figura 1). Este simulador no permite modificar el código fuente por lo tanto no permite la modificación del modelo. El programa desarrollado presenta un entorno fácil de usar por operadores, ingenieros o jefes de plantas con el fin de simular y estudiar el efecto de diversas variables sobre los procesos biológicos. Desass es también utilizado como software educativo en el estudio de la eliminación de nutrientes, configuración y diseño de plantas de tratamiento.

Este simulador permite calcular el rendimiento en estado estable o transitorio de diversas unidades de tratamiento: decantadores primarios (con procesos de fermentación), tanques fermentadores (generación de materia orgánica fácilmente biodegradable), reactores biológicos (anaerobios, anóxicos y aerobios) para la simulación de procesos como eliminación de nitrógeno (nitrificación-desnitrificación) y eliminación de fosforo. También permite simular la etapa de deshidratación de fangos y estudiar la influencia de esta carga contaminante sobre el proceso (Ferrer et al., 2008; Ferrer et al., 2005; Ferrer et al., 2004).

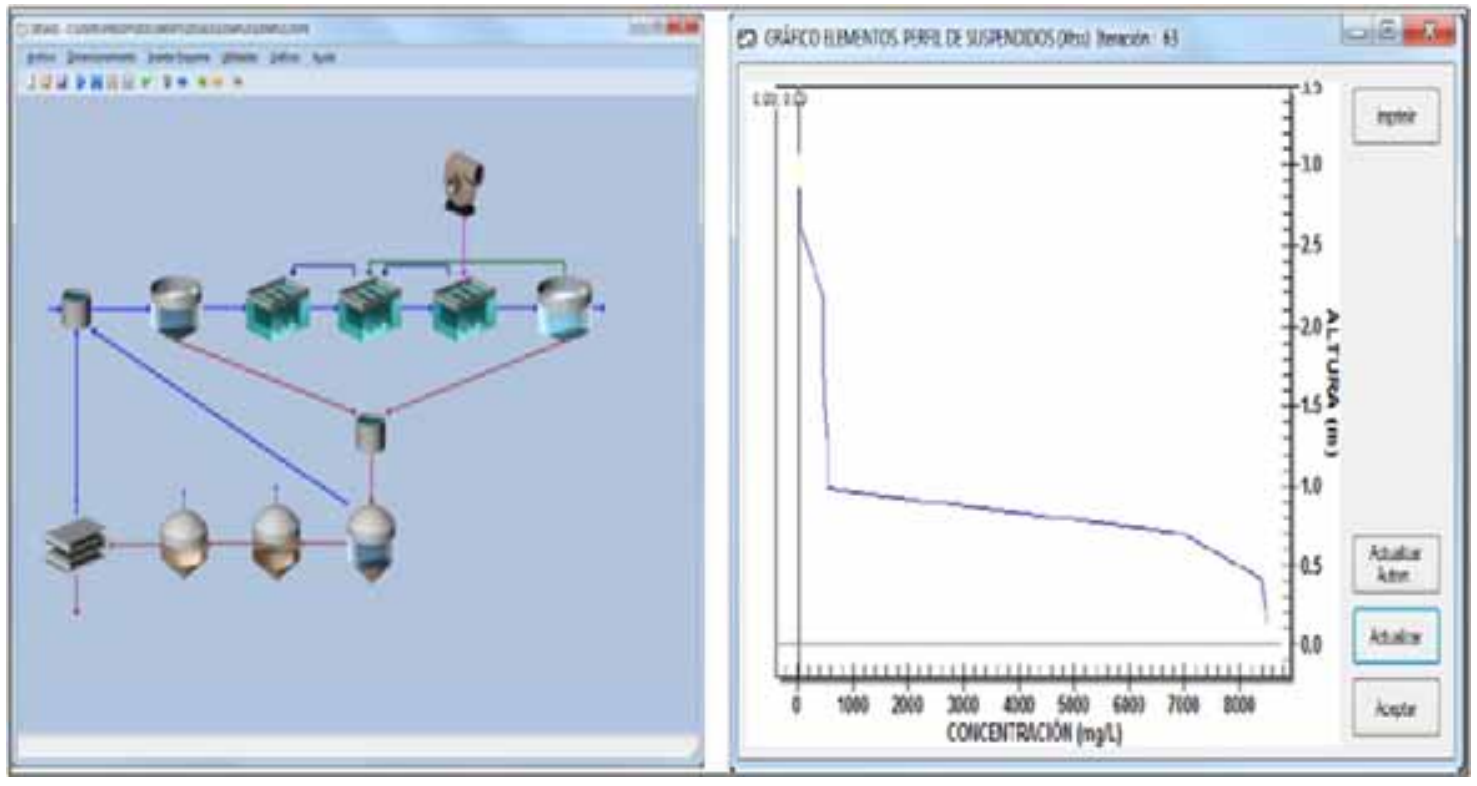

Figura 1. Entorno gráfico: DESASS. 
El modelo matemático implementado en DESASS es el BNRM1 (Seco et al., 2004). Este modelo considera los procesos físicos, químicos y biológicos más importantes y que tienen lugar en una planta de tratamiento de agua residual. Los procesos físicos que se incluyen son: sedimentación y clarificación (Takacs et al., 1991), elutriación del fango (Ribes et al., 2002), transferencia de gas-liquido, interacciones químicas acido-base (Serralta et al., 2003; Durán, 2014) y precipitación de fosforo, además incluye los procesos descritos en el modelo ASM2 (eliminación biológica de materia orgánica, nitrógeno, fósforo, acidogénesis, acetogénesis y metanogénesis). El efecto de la temperatura en los procesos se considera en este modelo usando la ecuación general de Arrhenius.

\section{Aplicaciones}

Diseño: DESASS permite el cálculo del estado estacionario para las condiciones de invierno y de verano simultáneamente, ofreciendo así una fácil comparación entre cada una de las estaciones.

Simulación: En régimen transitorio el usuario simulará la evolución de la planta teniendo en cuenta la variación diaria en la entrada. También se puede realizar una simulación con entrada constante para ver cómo evoluciona el sistema frente a alguna modificación en las condiciones de operación (García, 2012)

Control: DESASS permite diseñar estrategias para controlar el oxígeno disuelto, el amonio y el nitrato. Una característica muy importante es que los algoritmos de control se pueden implementar en una planta real sin más que sustituir los datos que actualmente se obtienen del modelo por las señales enviadas por los aparatos de medida.

\subsection{BIOWIN}

BIOWIN fue desarrollado por Peter Dold, profesor en la Universidad de McMaster en Canadá, implicado en el desarrollo de modelos y control de procesos de fangos activados desde 1978. Su origen se establece en un programa desarrollado en fortran para un modelo elaborado en la Universidad del Cabo por varios investigadores conocidos en el tratamiento de agua residual, entre los que se encontraban Marais, Ekama y Dold. Este simulador contiene un modelo incorporado que no puede ser modificado por el usuario, cada unidad de proceso disponible está establecida y cada una de ellas tiene su modelo disponible

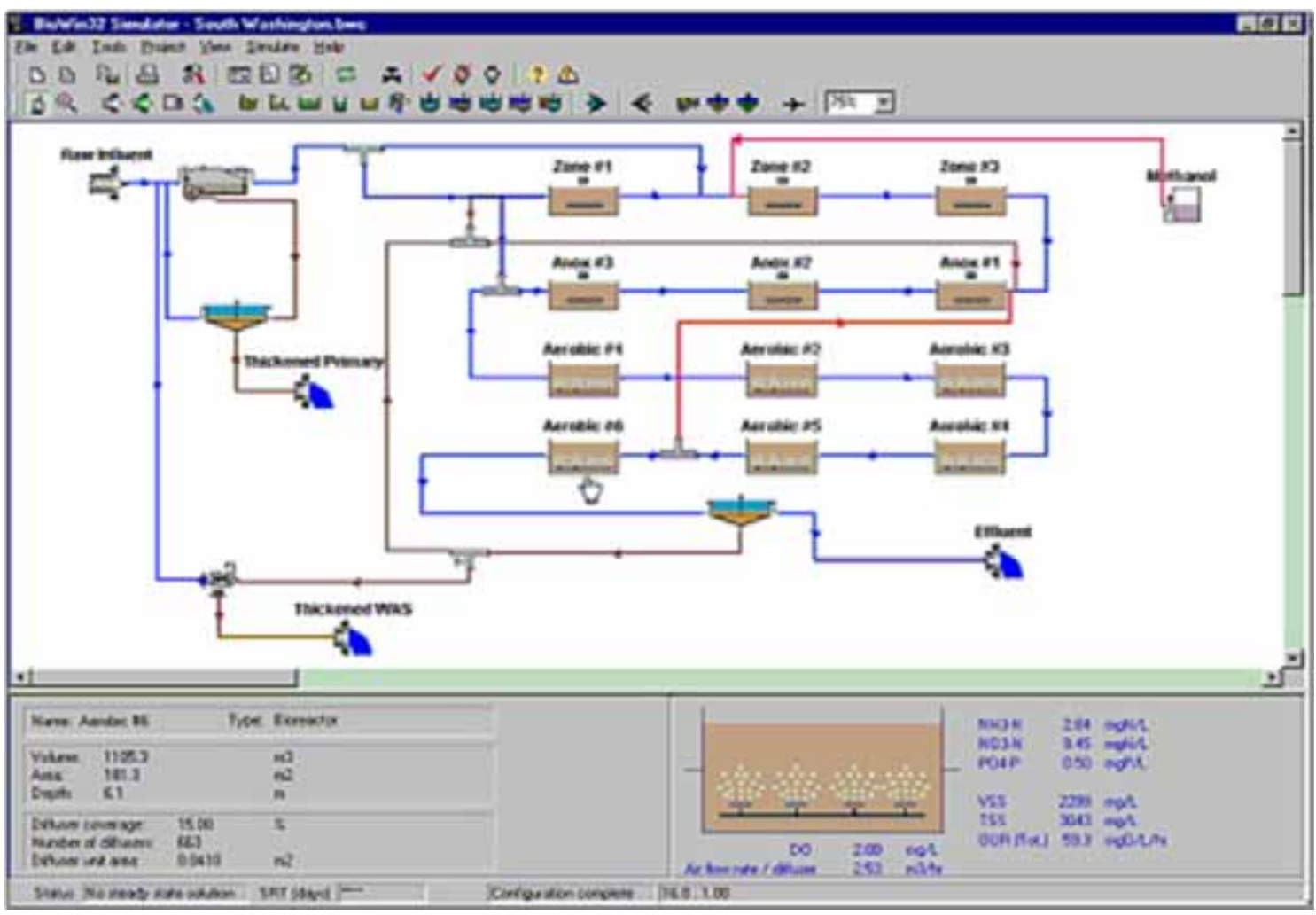

Figura 2. Entorno gráfico: Biowin. 
(figura 2). El modelo permite simular y diseñar, biorreactores, reactores (SBR), decantadores (clarificador), digestores anaerobios y elementos del digestor anaerobio (Gustaf et al., 2000; Portillo et al., 2012; Vitanza et al., 2015).

Este simulador contiene un modelo completo que incorpora 47 procesos que están descritos en el Manual Models Used In BioWin. La caracterización de la materia carbonácea se hace en términos de DQO. Sin embargo se hacen los cálculos pertinentes para permitir al usuario utilizar DBO, como filtrada o total, de 5, 7 o 20 días. Esta conversión se basa en tasas de degradación de diferentes componentes. Para la digestión anaerobia se utiliza otro modelo. En él se dan procesos que aparecen en el de fangos activados pero en condiciones diferentes, como la hidrólisis, fermentación y regulación de $\mathrm{pH}$. Este programa permite simular el comportamiento dinámico de plantas de tratamiento de efluentes tanto municipales como industriales (Mike et al., 2009; Contreras Barrera et al., 2010).

Posee los modelos más completos, flexibles y precisos del mercado, permitiéndole al usuario evaluar entre otros procesos: Remoción de material carbonáceo (DQO, DBO), nitrificación, desnitrificación, remoción biológica de fósforo, fermentación y digestión (aeróbica y anaeróbica), producción de metano, interacción de procesos físico-químicos (precipitación de fósforo y estruvita), sedimentación primaria y secundaria, espesamiento y deshidratación de lodos y desinfección de efluentes (Liwarska-Bizukojc et al., 2010; Baquero et al.,2014).

\subsection{EFOR}

El software de simulación EFOR fue desarrollado a finales de los ' 80 y principios de los '90 por un grupo de consultores daneses en colaboración con la Universidad Técnica de Dinamarca y VKI (posteriormente DHI). Desde 1991 se distribuyó por la compañía EFOR Aps propiedad de Krüger y Emolet Data. En el 2000 se integra el producto en la línea de software de DHI. $\mathrm{Su}$ entorno fue desarrollado en Microsoft para Windows (Gustaf et al., 2000).

La utilización de EFOR está estructurada en 5 actuaciones básicas que permiten establecer la información necesaria para ejecutar la simulación y una sexta donde se van a reflejar la información de salida, estas seis fases necesarias para ejecutar una simulación con EFOR son:
La sección de diseño: es un entorno gráfico que sirve para establecer la estructura física de la EDAR con la que se puede trabajar.

La sección de operación: se ejecutan lazos de control basados en sensores y dispositivos de control parecidos a los que existen en una EDAR. Se pueden generar también ciclos operativos permitiendo simular y controlar los proceso de una planta de tratamiento (Pedersen, 1992).

El editor de caracterización del influente: Mediante hojas de datos se introducen los datos de caudales y composición del influente.

El editor de temperaturas: Simular al editor anterior, permite determinar la temperatura en los tanques.

El modelo matemático: El programa permite seleccionar varios modelos matemáticos basados o derivados en los ampliamente difundidos por la IWA.

El gestor de gráficos y tablas: Antes de ejecutar la simulación es necesario definir como se quiere ver la información. Los resultados se pueden ver bien de forma dinámica, mientras se ejecuta la simulación o bien de forma estática.

\subsection{GPS-X}

GPS-X (General Purpose Simulator) ha sido desarrollado por Hydromantis, Inc. empresa consultora canadiense fundada en 1985, especializada en la ingeniería ambiental. La primera versión data de 1991 y actualmente se distribuye la 4.1. Se puede descargar de Internet la versión completa (funciona como demo) así como abundante material escrito sobre la aplicación (Gustaf et al., 2000; Pons et al., 1999).

La simulación se puede desarrollar en estado estacionario y simulación dinámica con presentación en tiempo real de las gráficas de evolución de las variables seleccionadas (figura 3). Permite generar esquemas de la planta piloto, soporte y conversión de unidades, desarrollo matemático mediante análisis numérico, simulación de plantas sin límite de tamaño, comunicación directa con office y matlab, comparación de datos reales y simulados, informes estadísticos y simulación de controladores (Chan et al., 2003; Nasr et al., 2011 ).

Los modelos biológicos utilizados por el simulador comprenden: ASM1 para la transformación y eliminación del carbono y del nitrógeno. ASM2d para la transformación y eliminación del carbono, del nitrógeno y del 


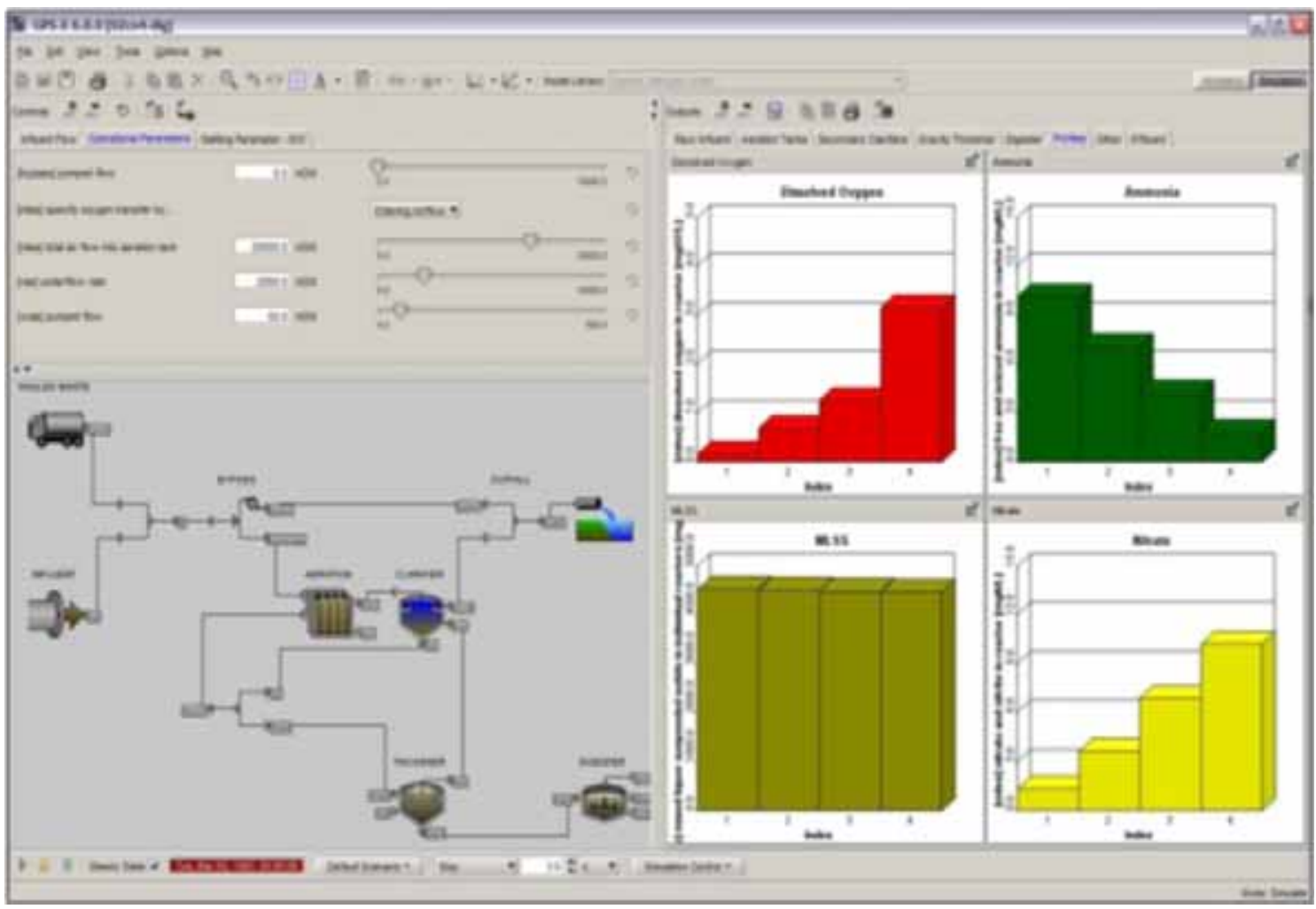

Figura 3. Entorno grafico: GPS-X.

fósforo. ASM3 para la transformación y eliminación del carbono y del nitrógeno.

Mantis es una versión creada por Hydromantis a partir del modelo ASM1 para describir de una forma más precisa la desnitrificación aerobia. 2-Step Mantis: Modificación del modelo Mantis con nitrificación en dos fases y New General: ASM1 ampliado a la eliminación del fósforo (Modelo de Peter Dold publicado en 1997). Existen también una versión que utiliza los modelos anaeróbios, como el caso del Basic anaerobic model (Andrews-Barnett) que incluye: destrucción de sólidos suspendidos volátiles, formación de ácidos grasos volátiles, metano, dióxido de carbono, $\mathrm{pH}$ y toxicidad por amonio. El modelo más conocido es el ADM1 (IWA) que incluye: destrucción de sólidos suspendidos volátiles, cambios en los ácidos grasos volátiles (acetato, propionato, valeriato y butirato), producción de biogás (metano, hidrógeno y dióxido de carbono), pH, inhibición (amoniaco, ácido acético e hidrógeno).

\subsection{SIMBA}

El sistema de simulación SIMBA permite la simulación de redes de alcantarillado (procesos de precipita- ción escorrentía, modelización hidrodinámica e hidrológica), plantas de tratamiento de aguas residual, tratamiento de lodos y simulación de agua superficial (flujo de contaminantes en ríos). Todos los componentes necesarios para un análisis en profundidad de los subsistemas constituyentes, incluyendo sus interacciones, ya están disponibles en el mismo simulador (Gustaf et al., 2000).

Puede aplicarse para una amplia variedad de tareas en la práctica de la ingeniería, investigación y en la educación (Erber et al., 2002). Permite el diseño de plantas de tratamiento de agua residual incluyendo el control de procesos, optimización y funcionamiento de plantas existentes (figura 4). Además incorpora un módulo de análisis de la gestión de agua de proceso, tratamiento de lodos y consumo energético (Alex 2009; Handbuch et al., 2001).

SIMBA es un simulador de plantas de tratamiento desarrollado bajo un entorno de Matlab/simulik con una completa biblioteca de bloques. Este modelo permite la modificación e interacción con otros modelos. Posee un entorno grafico como el que se muestra en la figura 5 . 


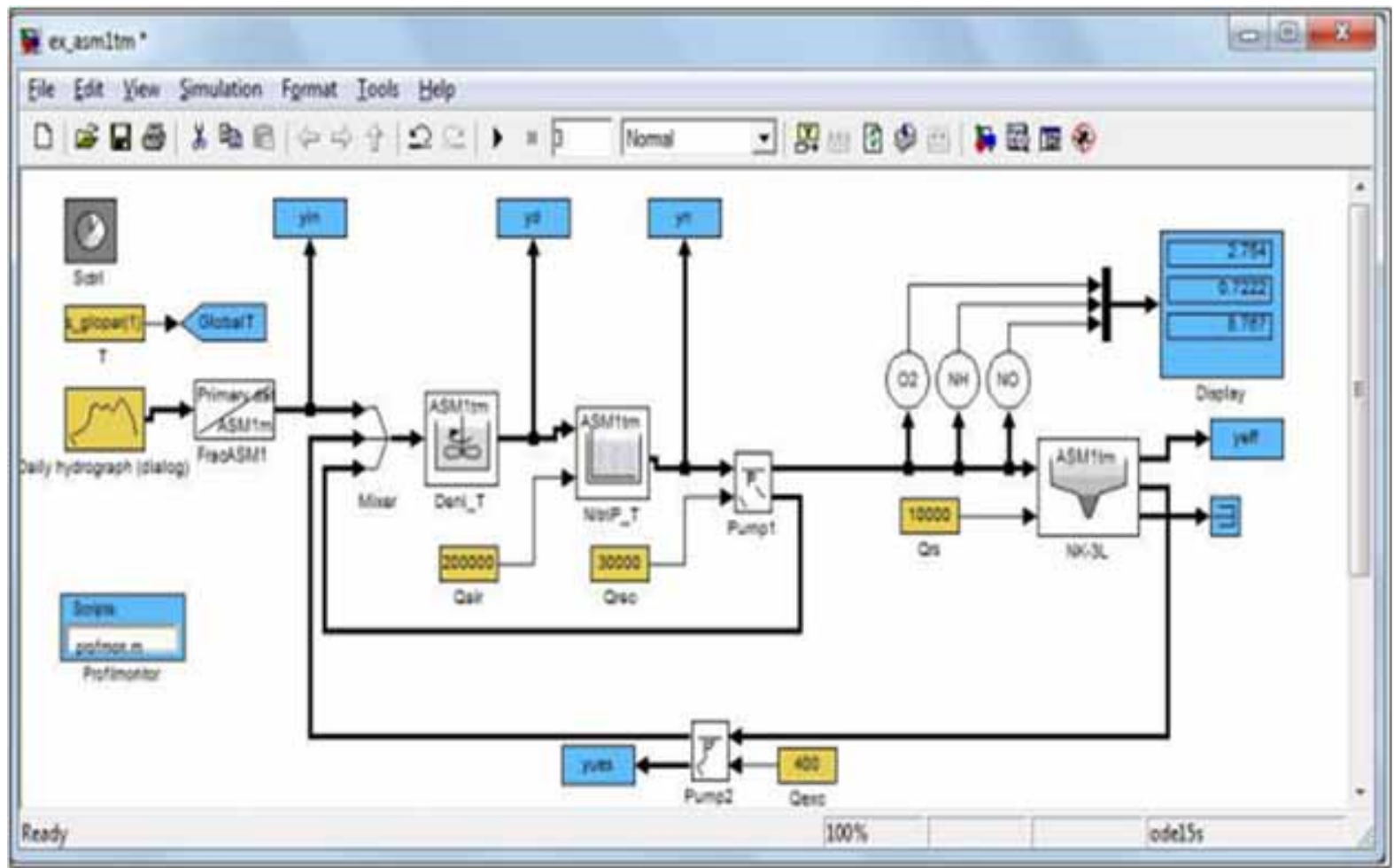

Figura 4. Entorno gráfico: SIMBA. .

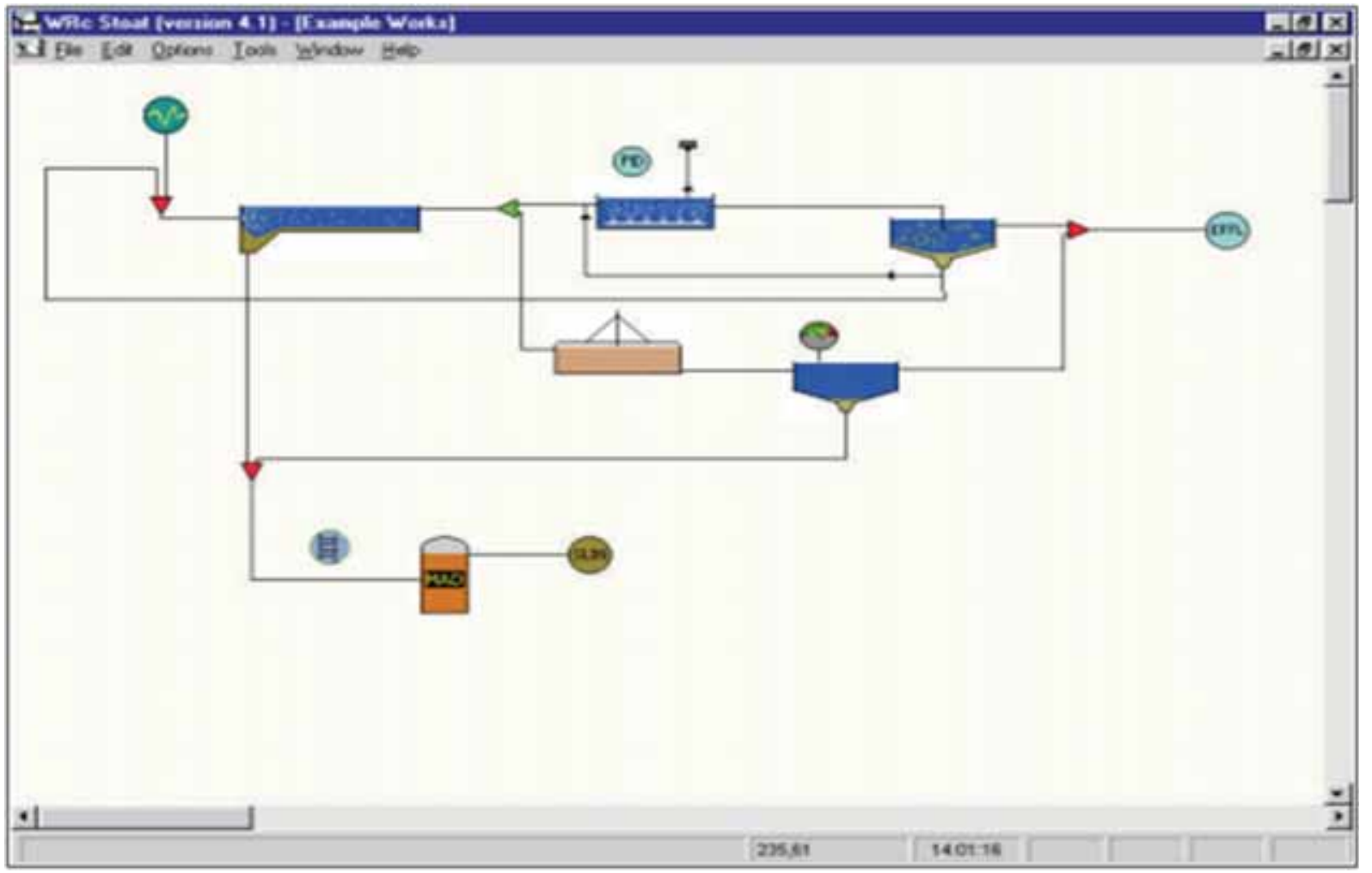

Figura 5. Entorno gráfico: STOAT. 


\subsection{STOAT}

Esta aplicación de simulación dinámica de estaciones depuradoras de aguas residuales ha sido desarrollada en el Reino Unido por WRc (Water Research Centre). STOAT se desarrolló en 1988 como parte del Programa de Gestión de la Contaminación Urbana en el Reino Unido. En 1994 se lanzó la primera versión siendo la actual la 4.2. STOAT forma parte de un conjunto de simuladores de WRc, junto con OTTER empleado para simulación de estaciones de tratamiento de agua potable y Plan-it para evaluar procesos de tratamiento de aguas residuales (Gustaf et al., 2000).

Este software fue diseñado para simular dinámicamente el rendimiento de una planta de tratamiento de aguas residuales (Sarkar et al., 2010). Puede ser utilizado para simular los procesos de tratamiento individuales o las obras completas de tratamiento, incluyendo el tratamiento de lodos, los procesos y las importaciones de tanques sépticos. El modelo permite al usuario optimizar la respuesta a los cambios en las cargas del afluente o variaciones importantes en las condiciones de operación del proceso (figura 5).

Dentro de las características más importantes de esta aplicación es que permite modelar el espectro completo de tratamiento de aguas residuales, línea de agua, línea de fangos, diferentes tipos de influentes, red de saneamiento previa (de forma simplificada). Se puede emplear de forma conjunta con programas de modelización de redes de saneamiento y ríos, posee un entorno grafico fácil de usar e incluye los modelos más utilizados de la IWA(ASM1, ASM2d y ASM3).

Permite diseñar modelos propios a partir de los existentes y admite distintas configuraciones para los reactores biológicos. Tiene modelos basados en DBO y DQO para la mayor parte de los procesos de tratamiento primario, secundario y terciario, incluye secado térmico de fangos e incineración (Stokes et al., 1997a; Stokes et al., 2000).

\subsection{WEST}

WEST (Word wide Engine for Simulation, Training and Automation) ha sido desarrollado por la empresa HEMMIS N.V. de Kortrijk (Bélgica). La primera versión de WEST se empezó a desarrollar en los años 90 en colaboración con la Universidad de Gante, departamento de matemáticas aplicadas, biometría y control de procesos (Gustaf et al., 2000).
La aplicación se estructura de la siguiente manera: la base de modelos (modelbase) o conjunto de ficheros de texto que contienen la descripción matemática de los procesos. Su orientación es eminentemente científica y el entorno gráfico de usuario (GUI) que posee, facilita el trabajo y una orientación eminentemente práctica (Mhlanga et al., 2009; Sochacki et al., 2009; Rey Gosálbez et al., 2007). Además contiene un completo motor de modelado y simulación. Emplea los modelos de la IWA(ASM1, ASM1T, ASM2, ASM2d, ASM2Dt y ASM3).

Otro aspecto importante en este software es que tiene una estructura abierta que permite la modificación del usuario, realizar el análisis de sensibilidad, estimación de parámetros y análisis de escenarios (figura 6).

\subsection{EDAR 1.0}

Este programa desarrollado ya hace algunos años por el CEIT (Centro de Estudios Investigaciones Técnicas de Guipuzcoa) para Cadagua, es comercializado por ATM, (Asistencia Tecnológica Ambiental SA). Una sencilla concepción, desarrollado para ser ejecutado bajo Windows, con pocos requerimientos de sistema (figura 7).

Sus principales características permiten el diseño de plantas de tratamiento con eliminación de materia orgánica y nitrógeno para aguas residuales urbanas e industriales. Además, simular la operación de plantas a nivel industrial y escala piloto. Su entorno lo hace atractivo para entornos académicos o profesionales.

Algunas de las opciones que el software ofrece son el diseño de plantas de forma sencilla, simular estados dinámicos o estacionarios y el fraccionamiento de los parámetros químicos como la DQO.

\subsection{AQUASIM}

AQUASIM es un programa del tipo "orientado a objetos". El programa ha sido desarrollado en el Instituto Federal Suizo de Ciencia y Tecnología Ambiental (EAWAG). En la web www.aquasim.eawag.ch se encuentran disponibles un manual de usuario y un tutorial de la versión 2.0 (Reichert, 1998). En este programa la configuración espacial de un modelo se representa mediante compartimentos que se conectan mediante enlaces (diagrama de flujo). Dentro de los compartimentos (un reactor, o un río, o un lago, o simplemente una cámara de aireación, etc.) se producen fenómenos: procesos de transporte, procesos 


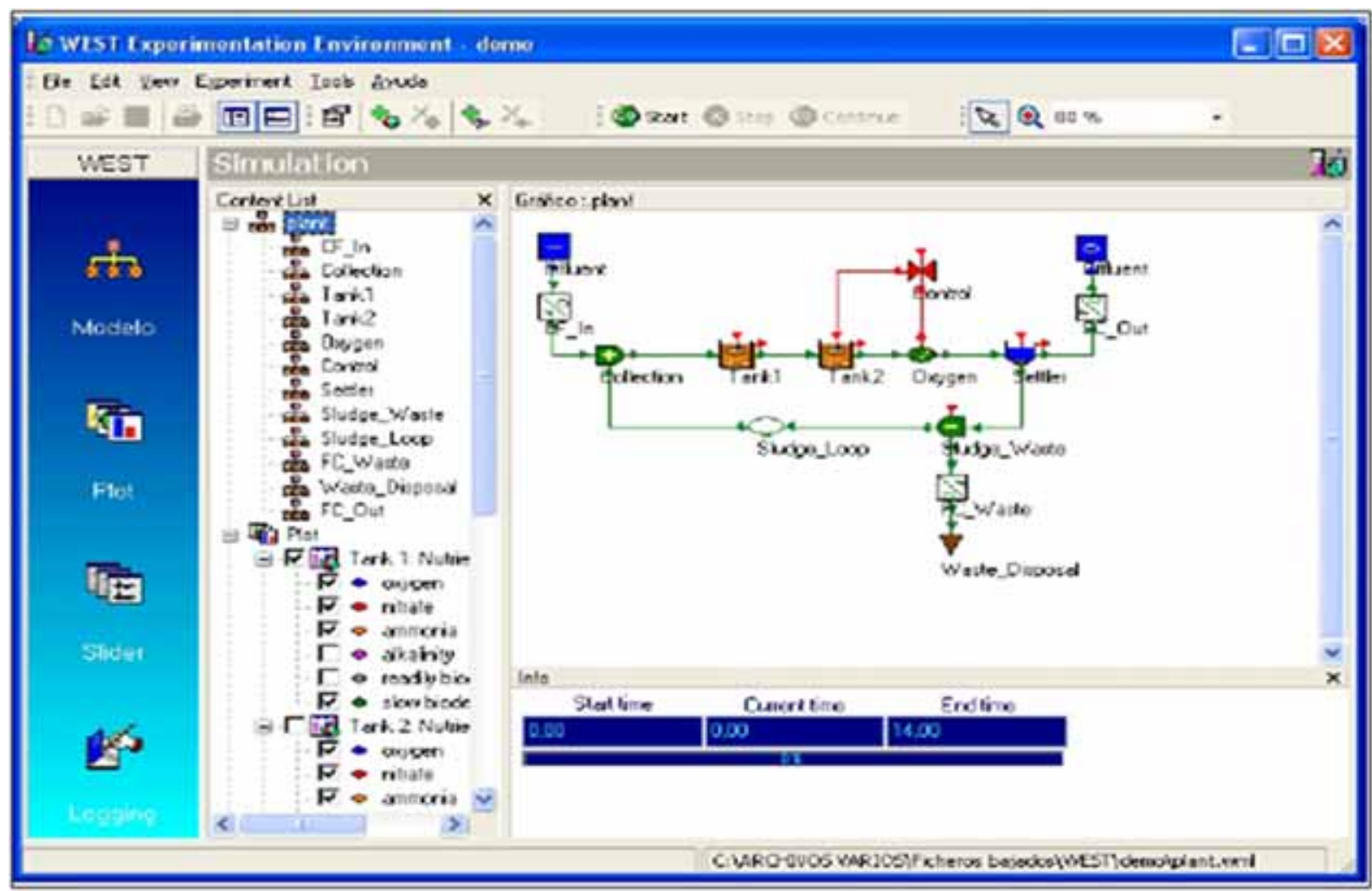

Figura 6. Entorno gráfico: WEST

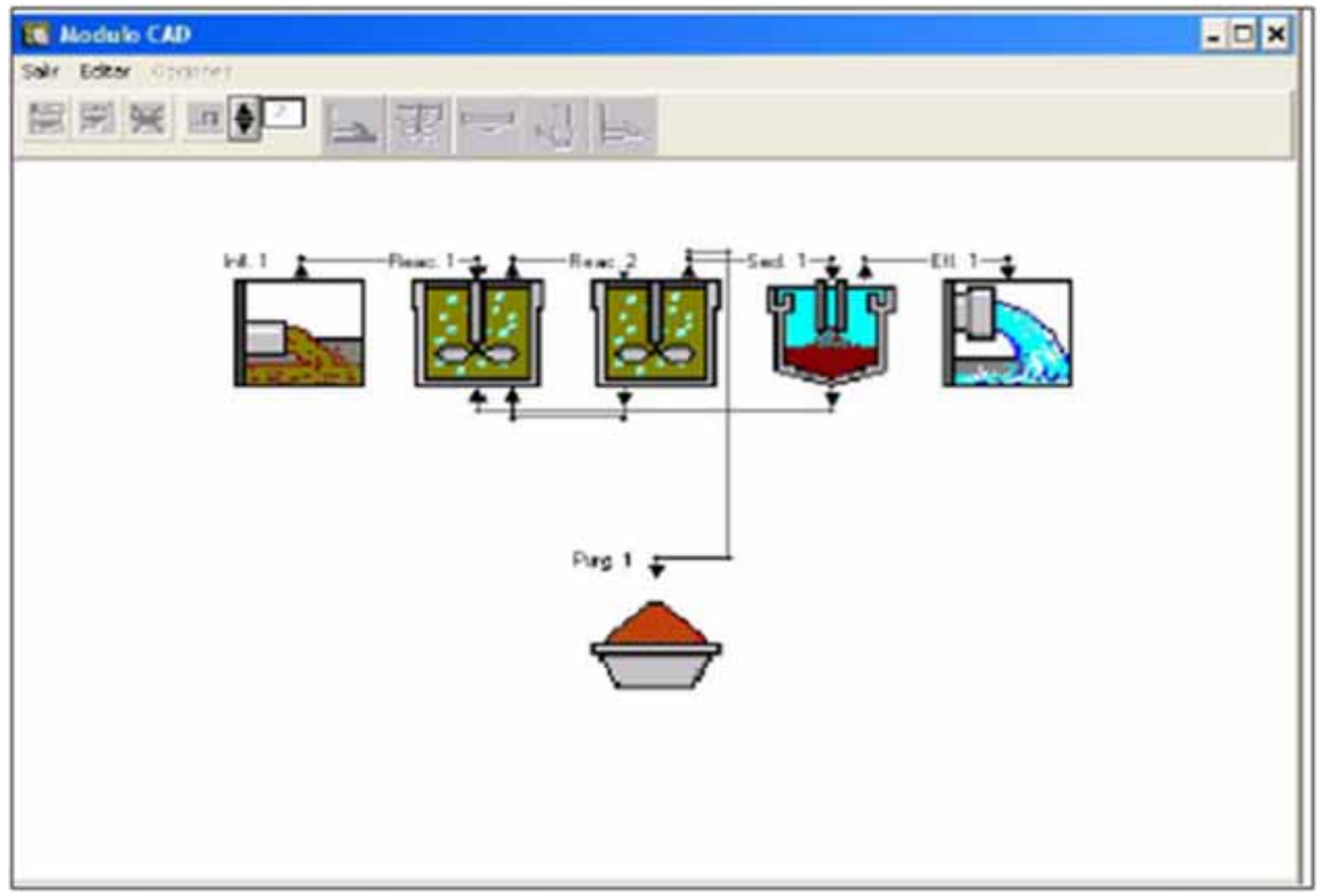

Figura 7. Entorno gráfico: Edar 1.0 
de reacción. El programa permite al usuario definir un número arbitrario de componentes o sustancias a ser modeladas y es extremadamente flexible en la formulación de los procesos de transformación. No sólo ofrece la posibilidad de realizar simulaciones de la evolución temporal del sistema especificado por el usuario, sino también métodos para la identificación de los parámetros del sistema modelizado (análisis de sensibilidad y estimación automática de parámetros), permitiendo estimar la incertidumbre de los resultados calculados.

Las presentaciones gráficas de simulaciones y de comparaciones con las mediciones son presentadas en tablas y gráficos que permiten el fácil análisis de datos (Figura 8). Utiliza un entorno gráfico con la definición de las variables y funciones de sensibilidad para varios cálculos. Luego de realizar un cálculo, su definición gráfica puede usarse para mostrar los resultados en pantalla, para escribir los resultados a un fichero o para su procesamiento externo con un programa adecuado (p.ej.: Excel).

\section{Conclusiones}

Los programas presentados anteriormente son una herramienta útil para el diseño, simulación y optimización de plantas de tratamiento de agua residual. Cada aplicación considera diversos procesos físicos y biológicos que deben ser evaluados para la utilización de los mismos.

Hasta el momento estas aplicaciones se han utilizado en diversos entornos con resultados satisfactorios. La principal característica de las aplicaciones mencionadas es el fácil manejo, lo cual permite observar por parte del personal técnico las consecuencias de modificar los criterios de operación en plantas existentes.

Este documento muestra las aplicaciones más utilizadas para el diseño y simulación de plantas de tratamiento de agua residual, basados en los modelos desarrollados por la IWA. Cada aplicación presenta diversas características siendo necesario estudiar cada

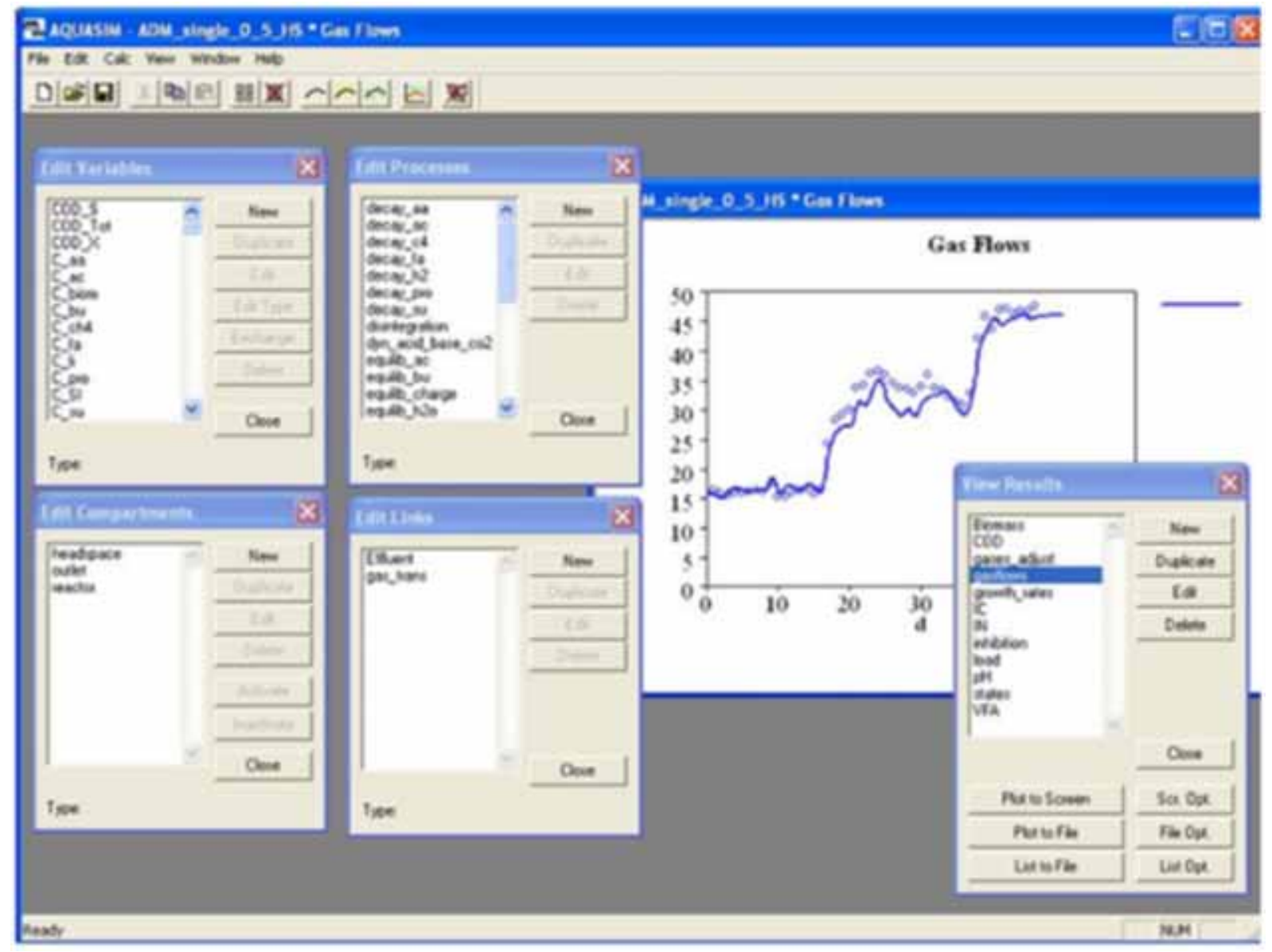

Figura 8. Entorno gráfico: AQUASIM. 
una para utilizar la más adecuada en el diseño o simulación de plantas de tratamiento de agua residual.

\section{Referencias bibliográficas}

1. Alex, J., 2009. Neue Entwicklungen im Abwasser simulations system SIMBA - Lagrange basierte Gewässergüte simulation., ifak-Institutskolloquium.

2. Anderson JS, Kim H, McAvoy TJ, Hao OJ., 2000. Control of an alternating aerobic-anoxic activated sludge system - Part 1: Development of a linearizationbased modeling approach. Control Eng. Pract., 8:271-278.

3. Ayesa Iturrate, E., Gran Gumbao, P., Salterain Ezquerra, A., \& Irizar Picón, I., 2007. Estrategias avanzadas de control automático para la eliminación de $\mathrm{N}$ en EDAR de Galindo. Tecnología del Agua, (284), 60-68.

4. Batstone D.J., Keller J., Angelidaki I., Kalyuzhnyi S.V., Pavlostathis S.G., Rozzi A., Sanders W. T. M., Siegrist H. and Vavilin V.A., 2002. Anaerobic Digestion Model No. 1. Scientific and Technical Report No. 13, IWA Publishing, London.

5. Baquero, A., Lara, J., Romero, C., Vargas, L., \& Martelo, J., 2014. Estudio de la tratabilidad del agua residual afluente a las futuras plantas de tratamientos salitre y canoas (tratamiento secundario) de la ciudad de Bogotá en plantas piloto con el sistema de lodos activados. Pontificia Universidad Javeriana.

6. Contreras Barrera, J. I., 2010. Modelación del sedimentador secundario en planta de lodos activados.

7. Copp JB., 2002. Experience with guidelines for waster characterization in The Netherlands. Technical report, The COST Simulation Benchmark: Description and Simulator Manual. Office for Official Publications of the European Community, ISBN 92894-1658-0, (Luxembourg).

8. Chan and Yue-ping., 2003. Simulation and analysis of biological wastewater treatment processes using GPS-X. The University of Hong Kong (Pokfulam, Hong Kong). Master of Science in Environmental Management.

9. Dircks K, Beun JJ, Van Loosdrecht MCM, Heijnen JJ, Henze M., 2001. Glycogen metabolism in aerobic mixed cultures. Biotechnol. BioEng., 73(2): 85-94.

10.Dold P, Ekama GA, Marais GVR., 1980. A general model for the activated sludge process. Progress in Water Technol., 12(6): 47-77.

11. Durán Pinzón, F., 2014. Modelación matemática del tratamiento anaerobio de aguas residuales urbanas incluyendo las bacterias sulfatorreductoras. Aplicación a un biorreactor anaerobio de membranas (Doctoral dissertation).

12.Erbe, V., Risholt, L. P., Schilling, W., \& Londong, J., 2002. Integrated modelling for analysis and optimization of wastewater systems-the Odenthal case. Urban Water, 4(1), 63-71.

13.Eriksson L, Hagbert P, Johansson E, R'annar S, Whelehan O, ${ }^{\circ}$ Astrom A, Lindgren T., 2001. Multivariate process monitoring of a newsprint mill. Application to modeling and predicting COD load resulting from deinking of recycled paper. J. Chemom., 15: 337-352.

14.Fang, F., Ni, B., Li, W., Sheng, G., \& Yu, H., 2011. A simulation-based integrated approach to optimize the biological nutrient removal process in a full-scale wastewater treatment plant. Chemical Engineering Journal, 174(2), 635-643.

15.Ferrer J., Seco A., Serralta J., Ribes J., Manga J., Asensi E., Morenilla J.J. y Llavador F., 2008. DESASS: a software tool for designing, simulating and optimising WWTPs. Environmental Modelling and Software, 23, 19-26.

16.Ferrer J., Morenilla J.J., Bouzas A. and Garcia-Usach F., 2004. Calibration and simulation of two large wastewater treatment plants operated for nutrient removal. This issue.

17.Ferrer J., Seco A., 2006. "DESASS 1.1 ®: Manual de usuario"; Universidad Politécnica de Valencia.

18.Ferrer J., Seco A., Ribes J., Serralta J., 2004. Biological Nutrient Removal Model No.1 (BNRM1); Universidad Politécnica de Valencia.

19.García Landero, I., 2012. Diseño de la Estación Depuradora de Aguas Residuales» EDAR Norte de Sevilla» mediante el software DESASS (Doctoral dissertation). 
20.Gujer W. and Henze M., 1991. Activated Sludge Modelling and Simulation, Wat. Sci. Tech., 24, Kyoto, pp 1011-1023.

21.Gujer W., Larsen T.A., 1995. The Implementation of Biokinetics and Conservation Principles in ASIM, Wat. Sci. Tech, 31, No. 2, 257-266.

22. Gernaey KV, Van Loosdrecht MCM, Henze M, Lind M, Jorgensen SB., 2004. Activated sludge wastewater treatment plant modeling and simulation: state of the art. Environ. Model. Software, 19: 763-783.

23.Gustaf Olsson and bob newell., 2000. Wastewater treatment systems: modeling, diagnosis and control, IWA publishing.

24.Grau-Gumbau, P., 2011. Nueva Metodología de Modelado Matemático Integral de las EDAR.

25.Handbuch, ifak e.V. Magdeburg., 2001. SIMBA 4.0 Simulation der biologischen Abwasserreinigung.

26.Hinken, L., Huber, M., Weichgrebe, D., \& Rosenwinkel, K. H., 2014. Modified ADM1 for modelling an UASB reactor laboratory plant treating starch wastewater and synthetic substrate load tests. Water research, 64, 82-93.

27. Henry M., 2003. Method for wastewater characterization in activated sludge modelin. Water environment research foundation.

28. Henze M, Grady Jr CPL, Gujer W, Marais GVR, Matsuo T., 1987. Activated Sludge Model No.1. Technical report, IWAPRC Scientific and Technical Report No.1 London (Great Britain).

29. Henze M, Gujer W, Mino T, Matsuo T, Wentzel MC., 1995. Activated Sludge Model No.2. Technical report, IWAPRC Scientific and Technical Report No. 2 London (Great Britain).

30.Henze, M., Gujer, W., Mino, T., and van Loosdrecht, M., 2000. Activated Sludge Models ASM1, ASM2, ASM2D and ASM3. London, UK: IWA Publishing.

31.Henze M., W. Gujer., T. Mino., and van Loosdrecht., 2000. Activated Sludge Models ASM1, ASM2, ASM2d and ASM3, IWA, Sci. and Tech. Report No. 9. IWA Publishing, London (2000).
32.Henze M., W. Gujer., T. Mino., T. Matsuo., M.C. Wenzel., and C.V.R. Marais., 1999. Activated sludge model no. 2d Water Sci. Technol., 39 (1) (1999), pp. $165-182$

33. Henze, M., 2008. Biological wastewater treatment: principles, modelling and design. IWA publishing.

34.Iturrate, E. A., \& Urcola, A. L., 2005. Optimización del diseño, operación y control de las EDAR utilizando modelos matemáticos y herramientas de simulación. Tecnología del agua, (266), 74-79.

35.Liwarska-Bizukojc, E., \& Biernacki, R., 2010. Identification of the most sensitive parameters in the activated sludge model implemented in BioWin software. Bioresource technology, 101(19), 72787285.

36. Martínez, J. J. M., Bonora, I. B., Basiero, J. A., Fajardo, V., Zorrilla, F., Lloret, R. M., \& Granell, P., 2008. Utilización de herramientas de simulación para definir la estrategia óptima de implementación del proceso de eliminación biológica de nitrógeno en la EDAR de Ontinyent. Tecnología del agua, 28(299), 53-61.

37. Morenilla, J. J., Bernácer, I., Lloret, R. M., Basiero, J. A., Zorrilla, F., Fajardo, V \& Granell, P., 2008. Utilización de herramientas de simulación para definir la estrategia óptima de implementación del proceso de eliminación biológica de nitrógeno en la EDAR de Ontinyent. Tecnología del Agua, (299), 53-61.

38. Mhlanga, F. T., Brouckaert, C. J., Foxon, K. M., Fennemore, C., Mzulwini, D., \& Buckley, C. A., 2009. Simulation of a wastewater treatment plant receiving industrial effluents. Water SA, 35(4), 447-454.

39. Metcalf y Eddy., 1991. Wastewater Engineering Treatment. Disposal, Reuse. 3a. Edition. USA. (Ingeniería en el tratamiento de aguas residuales. Disposición y reuso. Mc Graw-Hill International Editions. 3a. Edición. E.U.A.).

40.Mike M., Jamie G., Jason B., Chris W., Theresa B., and Don Howard., 2009. BioWin Modeling of a Three Reactor IFAS System. Proceedings of the Water Environment Federation 82ND Annual Technical Exhibition \& Conference, Orlando, FL, USA, October 10-14.

41.Nasr, M. S., Moustafa, M. A., Seif, H. A., \& El Kobrosy, G., 2011. Modelling and simulation of 
German BIOGEST/EL-AGAMY wastewater treatment plants-Egypt using GPS-X simulator. Alexandria Engineering Journal, 50(4), 351-357.

42. Olsson, G., B. Newell., 1999. Wastewater Treatment Systems: Modelling, Diagnosis and ControL, IWA.

43.Palma-Acosta, M. J., \& Manga Certain, J., 2011. Simulación de un sistema de fangos activados en discontinuo (SBR) para el tratamiento de aguas residuales con altos contenidos de nitrógeno.Revista Científica Ingeniería y Desarrollo, 18(18), 61-71.

44.Pedersen, J., 1992. Controlling activated sludge process using EFOR. Water Science \& Technology, 26(3-4), 783-790.

45.Pons, M. N., Spanjers, H., \& Jeppsson, U., 1999. Towards a benchmark for evaluating control strategies in wastewater treatment plants by simulation. Computers \& Chemical Engineering, 23, S403-S406.

46.Portillo, N. P., Melero, M. P. O., Campo, E. R., \& Izaguirre, J. P., 2012. Modelización y simulación de la EDAR de La Cartuja en Zaragoza, mediante el programa Bio Win 3.0. Tecnología del agua, 32(337), 42-49.

47. Reichert P., 1998a. AQUASIM 2.0 - User Manual, computer program for the identification and simulation of aquatic systems, Swiss Federal Institute for Environmental Science and Technology (EAWAG), Switzerland. ISBN 3-906484-16-5.

48. Reichert P., 1998b. AQUASIM 2.0 -Tutorial, computer program for the identification and simulation of aquatic systems, Swiss Federal Institute for Environmental Science and Technology (EAWAG), Switzerland. ISBN 3-906484-17-3.

49. Rey Gosálbez, H., Gracia Igelmo, M., Larrea Urcola, M. A., Morenilla Martínez, J. J., Bernácer Bonora, I., \& Santos Asensi, J. M., 2007. Optimización de la operación de la EDAR de Guarmar del Segura mediante la utilización de herramientas de simulación. Tecnología del Agua, (284), 38-46.

50.Ribes J., Ferrer J., Bouzas A. and Seco A., 2002. Modelling of an activated primary settling tank including the fermentation process and VFA elutriation. Environ. Technol., 23, 1147-1156.
51.Rivera-Salvador, V., Aranda-Barradas, J. S., Espinosa-Solares, T., Robles-Martínez, F., \& Toledo, J. U., 2009. El Modelo de Digestión Anaeróbica IWA-ADM1: una revisión de su evolución. Ingeniería Agrícola y Biosistemas, 1(2), 109-118.

52.Roeleveld PJ, Van Loosdrecht MCM., 2002. Experience with guidelines for waster characterization in The Netherlands. Water Sci. Technol., 45(6):77-87.

53.Sarkar, U., Dasgupta, D., Bhattacharya, T., Pal, S., \& Chakroborty, T., 2010. Dynamic simulation of activated sludge based wastewater treatment processes: Case studies with Titagarh Sewage Treatment Plant, India.Desalination, 252(1), 120-126.

54.Seco. A., Ribes. J.,Serralta. J., and Ferrer. J., 2004. Biological nutrient removal model No.1 (BNRM1). Water Science \& Technology. 50(6), 69-70.

55. Serralta J., Ferrer J., Borras L. and Seco A., 2003. A chemical model to calculate the $\mathrm{pH}$ in biological processes. Submitted to Water Research.

56. Smolders GJF, Klop JM, van Loosdrecht MCM, Heijnen JJ., 1995. Metabolic model of the biological phosphorus removal process: part I. Effect of the sludge retention time. Biotechnol. BioEng. 48: 222-233.

57. Sochacki, A., Knodel, J., Geißen, S. U., Zambarda, V., Miksch, K., \& Bertanza, G., 2009. Modelling and simulation of a municipal WWTP with limited operational data. In Proceedings of a PolishSwedish-Ukrainian Seminar (pp. 23-25).

58.Stokes, A. J., West, J. R., Forster, C. F., Kruger, R. C. A., De Bel, M., \& Davies, W. J., 1997a. Improvements to a stoat model of a full scale wastewater treatment works through the use of detailed mechanistic studies. Water science and technology, 36(5), 277-284.

59.Stokes, A. J., West, J. R., Forster, C. F., Kruger, R. C. A., De Bel, M., \& Davies, W. J.,1997b. Improvements to a STOAT model of a full scale wastewater treatment works through the use of detailed mechanistic studies. Water science and technology, 36(5), 277-284.

60.Stokes, A. J., West, J. R., Forster, C. F., \& Davies, W. J., 2000. Understanding some of the differences 
between the COD-and BOD-based models offered in STOAT. Water Research, 34(4), 1296-1306.

61.Takács, I., Patry, G.G. and Nolasco, D., 1991 A dynamic model of the clarification-thickening process. Wat. Res., 25(10), 1263-1271.

62. Van Loosdrecht M.C.M y Henze M., 1999. Maintenance, endogeneous respiration, lysis, decay and predation. Wat. Sci. Tech. 39 (1), 107-117.
63. Vitanza, R., Colussi, I., Cortesi, A., \& Gallo, V., 2015. Implementing a respirometry-based model into BioWin software to simulate wastewater treatment plant operations. Journal of Water Process Engineering.

64. Water Treatment Hanbook, 1991. (Manual de Tratamiento de Agua. Degremont 6a. Edition Vol. I y II. E.U.A.). 
